\title{
Port-Based Modeling and Optimal Control for a new Very Versatile Energy Efficient Actuator
}

\author{
Oscar Gerelli*, Raffaella Carloni** and Stefano Stramigioli**
}

\begin{abstract}
In this paper, we analyze in depth the innovative very versatile and energy efficient (V2E2) actuator proposed in Stramigioli et al. (2008). The V2E2 actuator is intended to be used in all kind of robotics and powered prosthetic applications in which energy consumption is a critical issue. In particular, this work focuses on the development of a port-based Hamiltonian model of the V2E2 and presents an optimal control architecture which exploits the intrinsic hybrid characteristics of the actuator design. The optimal control guarantees the minimization of dissipative power losses during torque tracking transients.
\end{abstract}

\section{INTRODUCTION}

Mobile robots and rehabilitation devices have gained a lot of interest in the research community during the last decade. For these systems, energy efficiency is of crucial importance because of the existing trade-off between the realization of light-weighted devices and the life-time of the power source. For this reason, research efforts have been spent in pursuit of the design of an ideal actuator, depicted in Fig. 1, which should behave as follows:

- the energy flows from the power supply (energy storage) to the load when an output positive work is required;

- when negative work is performed on the load, the energy is completely recovered, i.e. no energy is lost during the conversion;

- finally, no energy flows when the actuator is required to have a perfectly stiff or a free moving output shaft, and to deliver a static output torque.

The arrow through the actuator of Fig. 1 means that the amount of energy converted from the electrical to the mechanical domain can change, e.g. by varying the control input of the actuator. The energy conversion is usually achieved by means of DC motors. As analyzed by Kim et al. (2000), such devices show an high energy efficiency only in the low-torque high-speed region. However, many robotic applications should deliver high output torques even at low speed revolution requiring the use of either big and heavy motors or output gears with high gain ratios, which cause non-negligible dynamic effects and the lost of the system backdrivability.

In the literature, a certain amount of strategies for reducing energy consumption is already present. For example, compliant actuators with tunable series elastic elements in the actuation chain have been introduced, among others, by Pratt and Williamson (1995) to guarantee a safety

\footnotetext{
1 *gerelli@ce.unipr.it, Dipartimento di Ingegneria dell'Informazione, University of Parma, I-43100 Parma, Italy.

$2 * *\{$ r.carloni,s.stramigioli $\} @$ utwente.nl, IMPACT Institute, Faculty of Electrical Engineering, Mathematics and Computer Science, University of Twente, 7500 AE Enschede, The Netherlands.
}

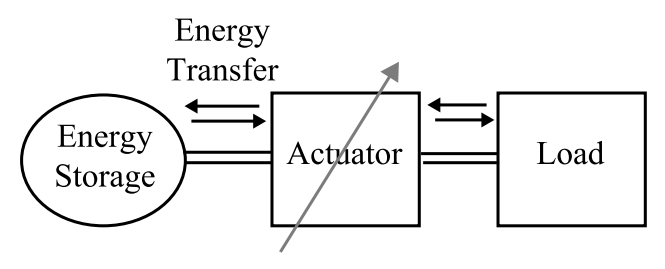

Fig. 1. Ideal actuator.

interaction between robots and their unstructured surrounding environment, and by Thorson et al. (2007); van Ham et al. (2005) to design biomimetic walking robots. Although series elastic elements can store energy, the compliant behavior can be changed only if energy is supplied.

A step forward in the direction of approaching some of the features of the ideal actuator of Fig. 1 have been proposed in Stramigioli et al. (2008), where a novel actuator concept has been described. This innovative very versatile and energy efficient actuator (V2E2) is depicted in Fig. 2. The main novelty lies in the use of an intermediate mechanical energy storage (MES) in conjunction with a mechanical Infinite Variable Transmission (IVT), see e.g. Mantriota (2002), connected to the system load.

In this paper we intend to present the port-Hamiltonian model of the V2E2 concept and an optimal control strategy which aims to minimize the overall power-losses during the tracking of a desired torque profile.

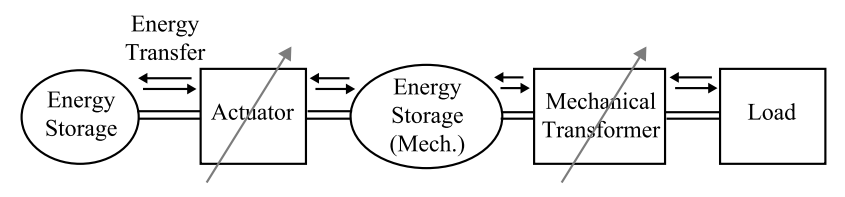

Fig. 2. The V2E2 actuator concept presented in Stramigioli et al. (2008).

\section{ACTUATOR MODEL}

\subsection{Behavior at a glance}

The V2E2 concept is made of a standard electrical drive, e.g. a DC motor, coupled to a mechanical energy storage. 
On the other side, the MES is connected to an IVT, used as the output interface toward the inertial load. The IVT, which represents the most complex component of the actuator, is a mechanical transformer whose transmission ratio can vary within a positive and a negative value $m \in\left[m_{\min }, m_{\max }\right]$, where $m_{\min } \in \mathbb{R}^{-}, m_{\max } \in \mathbb{R}^{+}$. The team of authors are currently working at the realization of an efficient IVT prototype with a ratio $m \in[-0.5,0.5]$.

In most practical situations, the actuator output torque will be provided by the stored energy in the MES, possibly modulated through the IVT; hence, the electrical drive has to compensate for frictions and the non-idealities of the system. In this work, a rotational spring has been used as mechanical energy storage in combination with a clutch on the output shaft of the electrical drive which prevent to unwind the spring from the motor side.

Due to the introduction of the cluch, the V2E2 actuator works in two operation modes: the unclutched case (UC), in which the clutch is not activated, and the clutched case (CC). Properly controlled, the UC configuration allows to mimic the first two requirements of an ideal actuator. In fact, when a positive work is performed on the load, energy is drained from the power source to increase the energy stored in the series spring. When negative work is done, the extra energy is not wasted, as in conventional actuators, but it can either be stored in the MES or it can be recovered to the power source by using the motor as a generator in conjunction with a reversible amplifier.

When the clutch is activated in the CC configuration, no energy injection occurs from the power supply since the output torque is entirely generated by the energy stored in the series spring. Therefore, the control action has only to modify the IVT ratio in order to compensate for power losses due to the IVT frictions. A smart IVT could be designed so to have friction coefficients comparable with the one of a simple fixed gear.

\subsection{The Hybrid evolution}

According to the discussion of the previous Section, it follows that the V2E2 actuator can have two different operation modes and, therefore, it can be modeled as an hybrid system Zefran et al. (2001); Goebel et al. (2004), in which the two discrete states correspond to the UC and the $\mathrm{CC}$ configurations. A high-level state transition diagram is reported in Fig. 3.

Assume the actuator is operating in clutched mode, i.e. the electrical drive is constrained at the zero angular velocity. Be $\tau_{s}$ the torque given by the spring due to its stored energy and be $\tau_{o}$ the output torque. To simplify the discussion, initially assume the IVT as a pure kinematic device. Therefore, the output torque $\tau_{o}$ can be obtained by simply setting the IVT transmission ratio $m$ according to the following equation

$$
m=\frac{\tau_{o}}{\tau_{s}}
$$

If the resulting value of $m$ is in the admissible range, i.e. $m \in\left[m_{\min }, m_{\max }\right]$, then the actuator can remain in the clutched state while assuring that the output torque $\tau_{o}$ is tracking a desired torque $\tau_{d}$. This condition fails any time the desired torque cannot be delivered by exploiting

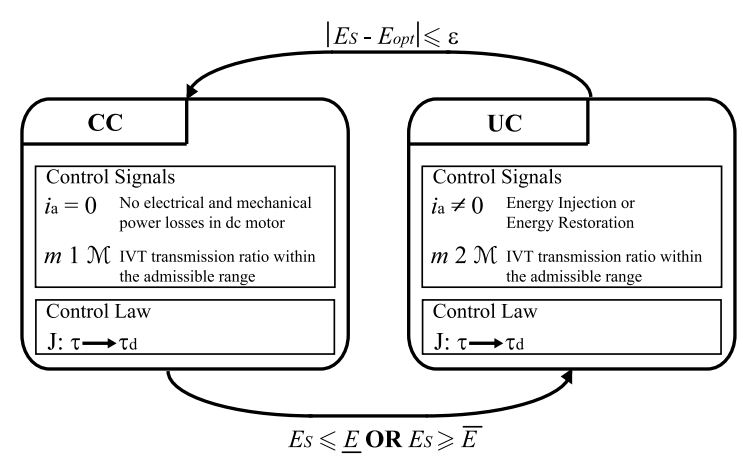

Fig. 3. State transition diagram for the hybrid model.

the IVT transmission ratio, i.e. when the energy $E_{s}$ stored in the MES is either too low or too high. In both cases, the clutch on the electrical drive has to be released. Note that, due to the system frictions, the energy conversion is not ideal and therefore, during the clutch phase, the spring energy tends to decrease. Since the spring should not be completely unloaded, see Stramigioli et al. (2008) for details, in Fig. 3 jumps are added when the stored energy becomes lower of a band guard value, i.e. $E_{s} \leq \underline{E}$. Analogously, to prevent the spring getting into a region of plastic deformation, a switch to the UC configuration is added when the energy exceeds an upper band guard value, i.e. $E_{s} \geq \bar{E}$, activating the electrical drive to recover the extra amount of stored energy. During the $\mathrm{CC}$ configuration, the current in the DC motor is fixed to $i_{a}=0$ since the electrical drive is decoupled from the MES and it does not influence the value of the output torque $\tau_{o}$. However, in order to preserve the energy accumulated in the elastic element, before the system state transition from $\mathrm{CC}$ to $\mathrm{UC}$ configuration, a suitable current $i_{a}$ is drained from the power source to generate, at the electrical drive output, a reaction force to avoid the spring to unwind, see Fig. 3.

In the UC state the output torque becomes a function of both the actuator variables, namely the motor current $i_{a}$ and the IVT ratio $m$. A degree of freedom is available to define an optimization control strategy and, in Section 3, a constrained control problem is formulated to minimize the system electromechanical power losses. If the transition to the UC configuration is caused by a low spring energy, i.e. $E_{s} \leq E$, the electrical drive has to be used as a motor to increase the amount of energy $E_{s}$. On the contrary, if $E_{s} \geq \bar{E}$, the electrical drive has to be used as a generator to recover the extra stored energy.

When $E_{s}$ reaches the value $E_{\text {opt }}$ defined by the optimization problem, the system can switch back to the CC configuration, i.e. the motor clutch is newly activated as shown in Fig. 3, and the output torque can again be statically delivered without continuously energy injections. Note that, in this case, the guard condition is $\left|E_{s}-E_{\text {opt }}\right|<\varepsilon$ in order to have robustness in the switch by introducing the threshold value $\varepsilon$.

\subsection{Actuator Model}

In this section, the model of the V2E2 actuator is presented using bond graphs and Port Hamiltonian formalism in or- 


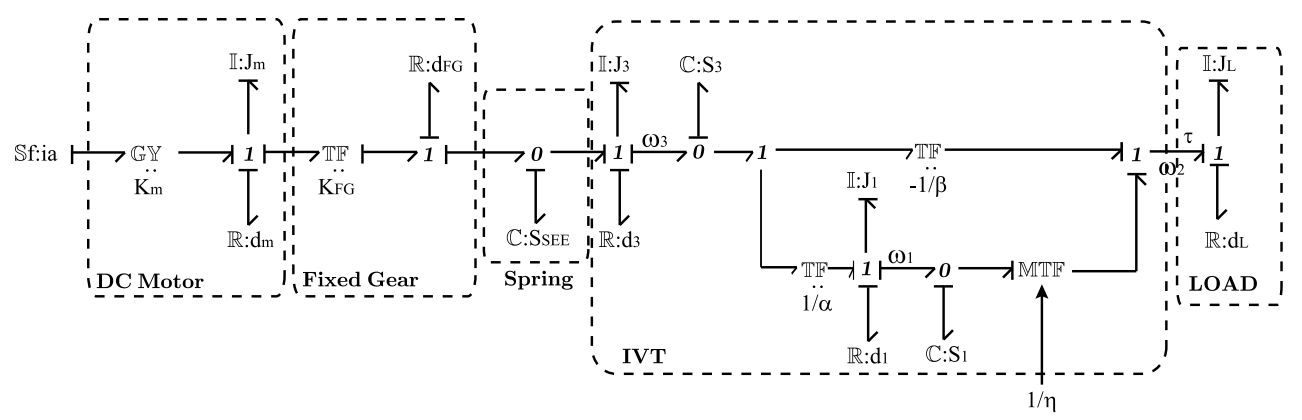

Fig. 4. Bond graph model of the actuator in its unclutched mode.

der to highlight the energetic relationships existing inside the system.

Bond graph models were early introduced by Paynter (1961), as a systematic graphical means of tracing systems energy flow. Any physical system can be modeled by properly interconnecting a set of simple elements, each of them characterized by a specific energetic behavior. Hence, differently from other graphical models, bond graph gives an insight on the energy interaction/flow between each elementary block, each of which is characterized by one or more power port. Power ports consist of a pair of conjugate variables, called effort and flow, whose product gives the power $\mathrm{P}$ instantaneously transferred through the port. Depending on the considered domain, effort and flow assume different meaning: for electrical ports they are, respectively, the voltage $v$ and the current $i$, while in the mechanical domain they represents the force $\tau$ and the velocity $\omega$ (see Karnopp et al. (1990) for more details).

The bond graph model of the V2E2 actuator, operating in the unclutched configuration, is shown in Fig.4. $K_{m}, J_{m}, d_{m}$ are the electrical drive parameters, i.e. the motor mechanical constant, inertia and friction coefficient, respectively. Analogously, $K_{F G}$ represents the transmission ratio of a small fixed gear connected to the electrical drive and $d_{F G}$ is its corresponding friction parameter. $S_{S}$ represents the elastic constant of the series spring used as MES and $J_{1}, J_{3}, d_{1}, d_{3}, S_{1}$ and $S_{3}$ are the inertias, friction coefficients and elastic couplings of the IVT shafts, respectively. According to its mechanical design, the IVT transmission is expressed as

$$
\left\{\begin{aligned}
\omega_{3} & =\eta \omega_{2} \\
\alpha \omega_{3} & =\beta \omega_{2}+\omega_{1}
\end{aligned}\right.
$$

where $\omega_{1}, \omega_{2} \in \mathbb{R}$ are the input and output angular velocities, $\omega_{3} \in \mathbb{R}$ is an internal angular velocity, $\alpha, \beta \in \mathbb{R}^{+}$ are constant ratio and $\eta \in \mathbb{R}^{+}$is the modulation input. Given (1), the IVT kinematic between the input and the output port is equal to

$$
\omega_{1}=(\eta \alpha-\beta) \omega_{2}=m \omega_{2}
$$

Equation (2) highlight that the modulation input $\eta$ allows for the continuous change of the IVT transmission ratio $m$, which therefore represents a control input to the system. In particular, if $\eta<\beta / \alpha$, the resulting value of $m$ is negative, otherwise if $\eta>\beta / \alpha$ the transmission ratio is positive. When $\eta=\beta / \alpha$ a decoupling between the input and the output port is realized.
Assumption 2.1. In the IVT model of Fig. 4 only mechanical frictions and inertias are considered, while it is assumed that any change of $\eta$ can be achieved instantaneously with no power losses.

Assumption 2.2. Without any loss of generality the load is modeled as a mass-damper system.

Given a bond graph model, the natural way to derive its analytically representation is by means of the portcontrolled Hamiltonian formalism. In particular, the hybrid port-controlled Hamiltonian model is described by the following set of differential and difference equations

$$
\begin{aligned}
\dot{x}(t)= & {\left[\mathcal{F}_{c}(x(t), u(t))-\mathcal{R}_{c}(x(t))\right]\left(\frac{\partial \mathcal{H}}{\partial x}(x(t))\right)^{T} } \\
& +G_{c}(x(t)) u_{c}(t)
\end{aligned}
$$

where the notation proposed in Haddad et al. (2003) has been used. The differential equation (3) is referred to as the continuous-time dynamics. Moreover, the skewsymmetric matrix function $\mathcal{F}_{c}(x, u)$ captures the internal hybrid system interconnection structure, the input matrix function $G_{c}(x)$ captures hybrid interconnections with the environment and the symmetric positive definite matrix function $\mathcal{R}_{c}(x)$ captures the hybrid system dissipation.

According to the model of Fig. 4, the system state vector is defined as $x:=\left(p_{m}, q_{S}, p_{1}, q_{1}, p_{3}, q_{3}, p_{L}\right)^{T} \in \chi \subseteq \mathbb{R}^{7}$, where $p_{x}$ indicates the generalized momentum related to inertia $J_{x}$, and $q_{x}$ is the generalized displacement related to elastic component $S_{x}$. The corresponding Hamiltonian function, representing the system total energy, is given by

$$
\begin{aligned}
\mathcal{H}(x):= & \frac{1}{2} \frac{p_{m}^{2}}{J_{m}}+\frac{1}{2} S_{S} q_{S}^{2}+\frac{1}{2} \frac{p_{1}^{2}}{J_{1}}+\frac{1}{2} S_{1} q_{1}^{2} \\
& +\frac{1}{2} \frac{p_{3}^{2}}{J_{3}}+\frac{1}{2} S_{3} q_{3}^{2}+\frac{1}{2} \frac{p_{L}^{2}}{J_{L}}
\end{aligned}
$$

while the system dynamics $\dot{x}=\left(\dot{p}_{m}, \dot{q}_{S}, \dot{p}_{3}, \dot{q}_{3}, \dot{p}_{1}, \dot{q}_{1}, \dot{p}_{L}\right)^{T}$ is expressed, after some algebraic manipulations, by means of the following equations

$$
\begin{aligned}
\dot{p}_{m} & =K_{m} i_{a}-\left(d_{m}+d_{F G} K_{F G}^{2}\right) \frac{\partial \mathcal{H}}{\partial p_{m}}-K_{F G} \frac{\partial \mathcal{H}}{\partial q_{S}} \\
\dot{q}_{S} & =K_{F G} \frac{\partial \mathcal{H}}{\partial p_{m}}-\frac{\partial \mathcal{H}}{\partial p_{1}} \\
\dot{p}_{1} & =\frac{\partial \mathcal{H}}{\partial q_{S}}-\frac{\partial \mathcal{H}}{\partial q_{1}}-d_{1} \frac{\partial \mathcal{H}}{\partial p_{1}}
\end{aligned}
$$




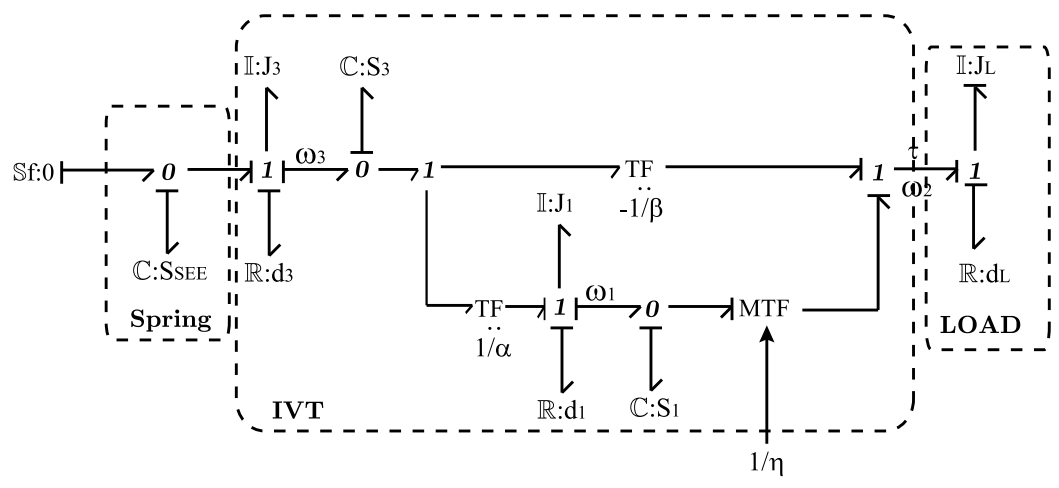

Fig. 5. Bond graph model of the actuator in its clutched mode.

$$
\begin{aligned}
\dot{q}_{1} & =\frac{\partial \mathcal{H}}{\partial p_{1}}-\alpha \frac{\partial \mathcal{H}}{\partial p_{3}}+\beta \frac{\partial \mathcal{H}}{\partial p_{L}} \\
\dot{p}_{3} & =\alpha \frac{\partial \mathcal{H}}{\partial q_{1}}-d_{3} \frac{\partial \mathcal{H}}{\partial p_{3}}-\frac{\partial \mathcal{H}}{\partial q_{3}} \\
\dot{q}_{3} & =\frac{\partial \mathcal{H}}{\partial p_{3}}-\eta \frac{\partial \mathcal{H}}{\partial p_{L}} \\
\dot{p}_{L} & =\eta \frac{\partial \mathcal{H}}{\partial q_{3}}-\beta \frac{\partial \mathcal{H}}{\partial q_{1}}-d_{L} \frac{\partial \mathcal{H}}{\partial p_{L}}
\end{aligned}
$$

Rearranging (5)-(11) into the matrix form (3), the interconnection and dissipation matrices of the hybrid portHamiltonian system are defined as

$$
\mathcal{F}_{c}(x, u):=\left[\begin{array}{ccccccc}
0 & -K_{F G} & 0 & 0 & 0 & 0 & 0 \\
K_{F G} & 0 & -1 & 0 & 0 & 0 & 0 \\
0 & 1 & 0 & -1 & 0 & 0 & 0 \\
0 & 0 & 1 & 0 & -\alpha & 0 & \beta \\
0 & 0 & 0 & \alpha & 0 & -1 & 0 \\
0 & 0 & 0 & 0 & 1 & 0 & -\eta \\
0 & 0 & 0 & -\beta & 0 & \eta & 0
\end{array}\right]
$$

$$
\mathcal{R}_{c}(x):=\operatorname{diag}\left(d_{m}+d_{F G} K_{F G}^{2}, 0, d_{3}, 0, d_{1}, 0, d_{L}\right)
$$

with input vector given by

$$
G_{c}(x):=\left[K_{m}, 0,0,0,0,0,0\right]^{T}
$$

It is not surprisingly that the interconnection matrix $\mathcal{F}_{c}(\cdot, \cdot)$ explicit depends on the control input $\eta$. Since $\mathcal{F}_{c}(\cdot, \cdot)$ captures the energy interconnections, a dependences from $\eta$ highlights the possibility to steer the power flows in the actuator by simply externally changing the IVT mechanical ratio.

When the output port of the electrical drive is latched, the system operates in its clutched configuration and the model simplifies as reported in Fig. 5. Two important differences can be evinced with respect to the UC configuration of Fig. 4:

- the V2E2 actuator reduces to a SISO system, being $\eta$ the only controlled input;

- the electrical drive dynamics does no longer influence the actuator behavior.

This allows to formulate the continuous dynamic in the clutched case

$$
\begin{aligned}
& \mathcal{F}_{c}(x):=\left[\begin{array}{ccccccc}
0 & K_{F G} & 0 & 0 & 0 & 0 & 0 \\
-K_{F G} & 0 & 0 & 0 & 0 & 0 & 0 \\
0 & 0 & 0 & 0 & 0 & 0 & 0 \\
0 & 0 & 0 & 0 & 0 & 0 & 0 \\
0 & 0 & 0 & 0 & 0 & 0 & 0 \\
0 & 0 & 0 & 0 & 0 & 0 & 0 \\
0 & 0 & 0 & 0 & 0 & 0 & 0
\end{array}\right] \\
& \mathcal{R}_{c}(x):=\operatorname{diag}\left(\left(d_{m}+d_{F G} K_{F G}^{2}\right), 0,0,0,0,0,0\right)
\end{aligned}
$$

with input vector given by

$$
G_{c}(x):=\left[K_{m}, 0,0,0,0,0,0\right]^{T}
$$

It is worth noting that, in the clutched state, the dissipative terms caused by the electrical drive dynamics are negligible, while the energy flows in the actuator can still be modified by acting on the IVT modulation input $\eta$. As explained in Stramigioli et al. (2008) and as previously remarked, the proposed actuator will operate in its clutched configuration in most practical operations, meaning that power losses are reduced, with respect to standard motors, simply by its mechanical design.

\section{CONTROL DESIGN}

The following sections are devoted to formalize the V2E2 actuator control architecture. According to Buss et al. (2000), we formulate two different dynamic controls depending on the current state of the hybrid model.

\subsection{Unclutched case}

When the actuator operates in the UC configuration, a degree of freedom is available to shape the output torque profile $\tau_{o}(t)$. More precisely, the output effort can be changed either by varying the transmission ratio $\eta$ or by varying the electrical motor output torque, through an energy injection from the main energy storage.

The available degree of freedom allows the definition of an optimal control problem. The goal is to track an output torque profile in finite time while minimizing the actuator power losses.

Problem 3.1. Given the dynamical system (3) described by matrices (12)-(14) and a desired output torque $\tau_{d}$, determine the control functions $i_{a}(t)$ and $\eta(t)$ such that

$$
\min _{i_{a}(t), \eta(t)} \int_{0}^{t_{f}} P_{\text {loss }}(t) d t
$$


where $P_{\text {loss }}(t)$ are the power losses during the transient, subject to the output torque tracking condition $\tau_{o}\left(i_{a}(t), \eta(t)\right)=\tau_{d}$ at the final time $t=t_{f}$ and such that spring energy conditions to preserve the UC condition (Fig. 3) are fulfilled.

It is worth noting that we are assuming the final time $t_{f}$ to be known in advance. Therefore, the transient time needed to generate the desired torque $\tau_{d}$ is not given as the outcome of the optimization problem but it is requested to be an input parameter, which value has to be designed according to the dynamic of the system.

At a high level, the actuator can be seen as a multi-bond system, described in Karnopp et al. (1990), consisting of a set of inputs, modeled as sources of flow $\mathbb{S}_{f}$, a set of dissipative elements $\mathbb{R}$ and energy storing blocks $\mathbb{C}$, as depicted in Fig. 6.

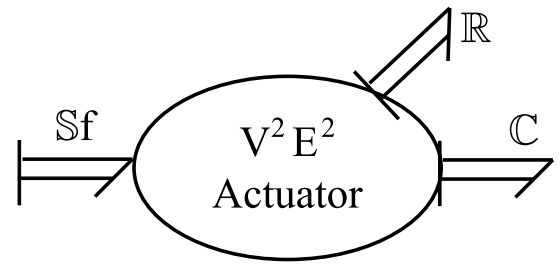

Fig. 6. High level representation of a port-Hamiltonian system.

The actuator power losses of the proposed model, shown in Fig. 4, are given by:

$$
P_{\text {loss }}(t)=R_{a} i_{a}^{2}+d_{m} \omega_{m}^{2}+d_{F G} \omega_{F G}^{2}+d_{1} \omega_{1}^{2}+d_{2} \omega_{2}^{2}+d_{3} \omega_{3}^{2}
$$

where time dependences are omitted for conciseness.

Proposition 1. Being the Hamiltonian (4) bounded from below, the system is passive.

Proof. Being $J_{m}, S_{S}, J_{1}, S_{1}, J_{3}, S_{3}, J_{\text {load }} \in \mathbb{R}_{>0}$, it is straightforward to observe the boundedness from below of equation (4). Moreover it holds that $\mathcal{H}(x)=0$ if and only if the state is $x=\underline{0} \in \mathbb{R}^{7}$.

Definition 3.2. A dynamical system defined by

$$
\begin{aligned}
& \dot{x}=f(x, u, t), \quad x(0)=x_{0}, \quad u(t) \in \mathcal{U} \\
& y=g(x, u, t)
\end{aligned}
$$

is said to be zero-state-detectable (ZSD) iff

$$
(u, y) \equiv(0,0) \Rightarrow x \rightarrow 0
$$

Proposition 2. System (3) defined by matrices (12)-(14) is zero-state detectable.

Proof. ZSD property can be simply proved by showing that when the system input, the output and the output derivatives till the $(n-1)$-order, with $n$ the state-space dimension, are set to zero also the system state converges to zero.

Proposition 3. The equilibrium point $x=0$ is a strict local minimum for the system (3).

Proof. Recall the Hamiltonian function defined in (4). It is straightforward to verify that

$$
(\nabla \mathcal{H})_{x}=\left[\frac{x_{1}}{J_{m}}, S_{S} x_{2}, \frac{x_{3}}{J_{1}}, S_{1} x_{4}, \frac{x_{5}}{J_{3}}, S_{3} x_{6}, \frac{x_{7}}{J_{\text {load }}}\right]
$$

and moreover that

$$
\left.\frac{\partial \mathcal{H}}{\partial x}\right|_{x=0}=0
$$

Being

$$
\frac{\partial^{2} \mathcal{H}}{\partial^{2} x}=\operatorname{diag}\left(\frac{1}{J_{m}}, S_{S}, \frac{1}{J_{3}}, S_{3}, \frac{1}{J_{1}}, S_{1}, \frac{1}{J_{\text {load }}}\right)>0
$$

it holds that $x=0$ is a strict local minimum for (3).

Proposition 4. Being $x=0$ a strict local minimum for (4) and being the system ZSD, the system is stabilizable.

Proof. see Van Der Schaft (1996).

To define the control strategy, the classical variational calculus is applied to the port-Hamiltonian system. The following theorem gives necessary conditions to find the optimal input $u^{*}$.

Theorem 3.3. Assume $u^{*}(t)$ is an optimal control input on $\left[t_{i}, t_{f}\right]$ for the nonlinear system (19) with respect to the control functional $I: \chi \times \mathcal{U} \rightarrow \mathbb{R}$

$$
I_{x}(u)=M\left(x\left(t_{f}\right), u\left(t_{f}\right)\right)+\int_{t_{i}}^{t_{f}} L(x(t), u(t), t) d t
$$

where $M(x, u)$ and $L(x, u, t)$ are continuously differentiable functions of each of their argument.

If $x^{*}(t)$ denotes the solution of differential equation (19) for the input $u^{*}(t)$, and the control Hamiltonian function is defined as

$$
\bar{H}(\lambda, x, u, t)=L(x, u, t)+\lambda^{T} f(x, u, t)
$$

then there exists $\lambda^{*}(t) \in\left(C^{1}\left[t_{i}, t_{f}\right]\right)^{n}$ such that

$$
\begin{aligned}
\frac{\partial \bar{H}}{\partial x} & =-\lambda^{*}(t) \\
\frac{\partial \bar{H}}{\partial u} & =0 \\
\lambda^{*}\left(t_{f}\right) & =\left.\frac{\partial M}{\partial x}\right|_{t=t_{f}}
\end{aligned}
$$

Proof. See, e.g., Agrachev and Sachkov (2004).

Equation (22) is called the adjoint differential equation and the function $\lambda^{*}(t)$ is called the co-state.

Remark 3.4. Theorem 3.3 expresses only necessary conditions for optimality. Therefore not every control $u^{*}(t)$ satisfying equations (22)-(24) for some co-state function $\lambda^{*}(t)$ need to be optimal.

The following convexity property gives a sufficient condition to determine the unique optimal control for a certain class of dynamic systems.

Proposition 5. If the control Hamiltonian (21) is convex with respect to the state $x$ and the control $u$, then conditions (22)-(24) are also sufficient for the uniqueness of the optimal control $u^{*}(t)$.

Based on (18), the cost function (20) is defined as follows:

$$
\begin{aligned}
M\left(x\left(t_{f}\right), u\left(t_{f}\right)\right)= & c_{1}\left(\left.\eta\left(t_{f}\right) \frac{\partial \mathcal{H}}{\partial q_{3}}\right|_{x_{f}}-\left.\beta \frac{\partial \mathcal{H}}{\partial q_{1}}\right|_{x_{f}}-\tau_{o}\left(t_{f}\right)\right)^{2} \\
L(x, u, t)= & c_{2}\left(\eta(t) \frac{\partial \mathcal{H}}{\partial q_{3}}-\beta \frac{\partial \mathcal{H}}{\partial q_{1}}-\tau_{o}(t)\right)^{2}+ \\
& c_{3}\left(R_{a} i_{a}(t)^{2}+x^{T} E x\right)
\end{aligned}
$$


where $\left(c_{1}, c_{2}, c_{3}\right) \in \mathcal{P} \subset\left(\mathbb{R}^{+}\right)^{3}$ are the cost parameters and where $E \in \mathcal{E} \subseteq \mathbb{R}^{7 \times 7}$ is the diagonal matrix defined as

$$
E=\operatorname{diag}\left(\frac{d_{m}}{J_{m}^{2}}+\frac{K_{F G}^{2} d_{F G}}{J_{m}^{2}}, 0, \frac{d_{1}}{J_{1}}, 0, \frac{d_{3}}{J_{3}}, 0, \frac{d_{2}}{J_{2}}\right)
$$

The Hamiltonian $\bar{H}(\lambda, x, u, t)$ then becomes

$$
\begin{aligned}
\bar{H}(\lambda, x, u, t)= & c_{2}\left(\eta \frac{\partial \mathcal{H}}{\partial q_{3}}-\beta \frac{\partial \mathcal{H}}{\partial q_{1}}-\tau_{d}\right)^{2} \\
& +c_{3}\left(R_{a} i_{a}^{2}+x^{T} E x\right) \\
& +\lambda^{T}\left[\left(\mathcal{F}_{c}-\mathcal{R}_{c}\right) \frac{\partial \mathcal{H}}{\partial x}+G_{c} u\right]
\end{aligned}
$$

Proposition 6. Equation (25) is convex with respect to both $x$ and $u$, being a composition of convex functions in $x$ and $u$.

Therefore, the solution of the two-point boundary value problem, defined by equations (22)-(24), is the constrained optimal control $u^{*}(t)$ which minimize the power injection during the transient phase of the torque tracking. According to the constraints introduced in Problem 3.1, a transition from the UC to the CC case, during the optimization time horizon $\left[t_{i}, t_{f}\right]$, never occurs: the clutched state is activated only when the system output reaches the desired torque $\tau_{d}$. Hence $u^{*}(t)$ is the actual optimal control and, for this reason, we have handled in a separated way the control strategy during the UC state and the clutched one, analyzed in the following section.

\subsection{Clutched case}

When the clutch is enabled, the desired output torque $\tau_{d}(t)$ is provided only by the force generated in the series spring, potentially modulated by the mechanical ratio $\eta$ applied to the IVT. In an ideal case, i.e. assuming that no power loss occur in the energy transfer, any desired output torque can be obtained by varying the control function $\eta(t)$ according to

$$
\tau_{o}(t)=\eta(t) \tau_{S}(t)
$$

where $\tau_{S}(t)$ is the torque generated by the series spring.

When also the IVT dynamics is considered, according to Fig. 5, output torque function is given by

$$
\tau(t)_{o}=-\beta \tau_{3}+\eta \tau_{1}
$$

and therefore the control input $\eta(t)$ needed for tracking $\tau_{d}(t)$ is equal to

$$
\eta(t)=\frac{\tau_{d}+\beta \tau_{3}}{\tau_{1}}=\frac{\tau_{d}+\beta \tau_{S}-f(x, \dot{x})}{\alpha \tau_{S}-g(x, \dot{x})}
$$

where

$$
\begin{aligned}
& f(x, \dot{x})=\beta\left[\tau_{J_{3}}+\tau_{d_{3}}\right], \\
& g(x, \dot{x})=\alpha\left[\tau_{J_{3}}+\tau_{d_{3}}\right]+\tau_{J_{1}}+\tau_{d_{1}} .
\end{aligned}
$$

\section{CONCLUSIONS AND FUTURE WORK}

This paper has addressed the problem of the portHamiltonian modeling and control for a new type of very versatile energy efficient actuator. It has been shown the hybrid nature of the system and a description of the actuator behavior in each working state has been given. Two different port-Hamiltonian model has been devised considering the constraint imposed on the internal electrical motor. An optimal control problem has been defined and the general Euler-Lagrange framework has been successfully applied to the port-Hamiltonian model.

The authors are currently working to the numerical simulations of the hybrid control strategy herein described.

\section{REFERENCES}

Agrachev, A. and Sachkov, Y. (2004). Control theory from a geometric viewpoint. Springer-Verlag.

Buss, M., von Stryk, O., Bulirsch, R., and Schmidt, G. (2000). Towards hybrid optimal control. Automatisierungstechnik, 9, 448459 .

Goebel, R., Hespanha, J., Teel, A., Cai, C., and Sanfelice, R. (2004). Hybrid systems: generalized solutions and robust stability. In Proc. IFAC Symposium Nonlinear Control Systems.

Haddad, W., Nersesov, S., and Chellaboina, V. (2003). Energybased control for hybrid port-controlled hamiltonian systems. Automatica, 39, 1425-1435.

Karnopp, D., Margolis, D., and Rosenberg, R. (1990). System dynamics: a unified approach. John Wiley \& Sons, Inc., NY.

Kim, J., Yeom, H., Park, F., Park, Y., and Kim, M. (2000). On the energy efficiency of cvt-based mobile robots. In Proc. IEEE International Conference on Robotics and Automation.

Mantriota, G. (2002). Performances of a series infinitely variable transmission with type i power flow. Mechanism and Machine Theory, 37, 579-597.

Paynter, H. (1961). Analysis and Design of engineering systems. MIT Press.

Pratt, G. and Williamson, M. (1995). Series elastic actuators. In Proc. IEEE/RSJ International Conference on Intelligent Robots and Systems.

Stramigioli, S., van Oort, G., and Dertien, E. (2008). A concept for a new energy efficient actuator. In Proc. IEEE/ASME International Conference on Advanced Intelligent Mechatronics.

Thorson, I., Svinin, M., Hosoe, S., Asano, F., and Taji, K. (2007). Design considerations for a variable stiffness actuator in a robot that walks and runs. In Proc. JSME Conference Robotics and Mechatronics.

Van Der Schaft, A. (1996). $L_{2}$ Gain and Passivity Techniques in Nonlinear Control. Springer-Verlag.

van Ham, R., Vanderborght, B., van Damme, M., Verrelst, B., and Lefeber, D. (2005). Maccepa: the actuator with adaptable compliance for dynamic walking bipeds. In Proc. Int. Conf. Climbing and Walking Robots and the Support Technologies for Mobile Machines.

Zefran, M., Bullo, F., and Stein, M. (2001). A notion of passivity for hybrid systems. In Proc. IEEE Conference on Decision and Control. 